\title{
La violence politique : repères et problèmes
}

\section{Philippe Braud}

\section{(2) OpenEdition \\ 12 Journals}

Édition électronique

URL : https://journals.openedition.org/conflits/406

DOI : 10.4000/conflits.406

ISSN : $1777-5345$

Éditeur :

CECLS - Centre d'études sur les conflits - Liberté et sécurité, L'Harmattan

Édition imprimée

Date de publication : 15 mai 1993

ISSN : 1157-996X

Référence électronique

Philippe Braud, «La violence politique : repères et problèmes », Cultures \& Conflits [En ligne], 09-10 |

printemps-été 1993, mis en ligne le 13 mars 2006, consulté le 07 décembre 2022. URL : http://

journals.openedition.org/conflits/406 ; DOI : https://doi.org/10.4000/conflits.406

Ce document a été généré automatiquement le 30 mars 2021.

\section{c) $(1) \Theta$}

Creative Commons - Attribution - Pas d'Utilisation Commerciale - Pas de Modification 4.0 International - CC BY-NC-ND 4.0

https://creativecommons.org/licenses/by-nc-nd/4.0/ 


\title{
La violence politique : repères et problèmes
}

\author{
Philippe Braud
}

1 "Quant à vouloir s'imposer à ses concitoyens par la violence, c'est toujours chose odieuse même si l'on se donne pour but de réformer des abus". Salluste ${ }^{1}$

2 La violence politique est le terrain d'élection des jugements de valeurs. A première vue il semblerait aujourd'hui que les condamnations morales, qui n'ont jamais manqué dans la pensée occidentale, soient devenues quasi unanimes avec la consolidation contemporaine des démocraties pluralistes. Il conviendrait pourtant de ne pas oublier un proche passé: les apologies sartriennes de la violence des colonisés ${ }^{2}$, les légitimations de la violence révolutionnaire (anarchistes et néo-léninistes) ou encore celles des "peuples supérieurs" (nationalismes chauvins, néo-nazismes...). Mais surtout il faut bien repérer ce raisonnement circulaire qui consiste à ne qualifier comme violences que les comportements jugés illégitimes ${ }^{3}$, réservant aux autres l'emploi d'un lexique euphémisant: coercition, contrainte, force, etc...C'est que les discours de stigmatisation globale de toute violence physique, même coûteuse en vies humaines ou en pertes matérielles, résistent mal à l'épreuve de certaines situations politiques. A chaud la guerre du Golfe (janvier 1991) a vu renaître des justifications, inattendues chez certains, du recours à la force ; de même, l'écho donné par les médias internationaux au traitement des prisonniers dans les camps de Bosnie (été 1992) a fait surgir des stigmatisations de l'inaction militaire dans des termes qui rappellent parfois expressément le précédent des puissances occidentales, passives devant la montée de l'hitlérisme. Si indiscutable que soit la nécessité sociale de cette approche éthique de la violence, ce n'est pas le terrain sur lequel se situe l'analyse de science politique. Il lui faut se défaire des habits du moraliste pour simplement tenter d'oeuvrer à l'élucidation de sa place exacte dans le fonctionnement d'un système politique : en l'occurrence, la démocratie pluraliste. Malgré les apparences peut-être, cette place demeure centrale quand bien même la démocratie politique repose sur le principe de sa forclusion. Des auteurs aussi différents que Machiavel, Hobbes ou Weber nous aident à penser son importance encore aujourd'hui. Pour le premier, on le sait, la menace, ou l'usage 
effectif, de la force est une ressource politique courante, et la condition dernière de l'efficacité de toutes les autres. Le second, focalisant son attention sur la violence de tous contre tous et l'angoisse primordiale qu'elle fait surgir, considère l'ordre politique né du Contrat social comme la réponse appropriée à ce défi permanent du chaos. Quant à l'auteur d'Economie et Société, il érige le monopole tendanciel de la violence légitime en principal critère du pouvoir politique de nature étatique. L'analyse contemporaine de science politique n'en a pas moins quelque mal à intégrer cette notion parmi ses principaux instruments conceptuels. Les raisons en sont variées. Des chercheurs, légitimement soucieux de mesure empirique, préfèrent aborder l'étude des conflits qui se prêtent davantage à la quantification ${ }^{4}$. Mais il existe bien entendu des conflits sans violences et, réciproquement, une violence peut être si intense qu'elle interdit toute réponse, empêchant l'émergence d'un antagonisme visible. Les principales difficultés à surmonter ont surtout un rapport avec le poids des jugements de valeurs présents dans un proche arrière-plan. C'est ainsi qu'Yves Michaud va jusqu'à récuser la possibilité même d'en donner une définition opératoire, critiquant sur ce point l'ambition des grandes enquêtes empiriques américaines des années soixante-dix ${ }^{5}$. Et de fait, il existe des conceptions proprement antagonistes de la violence politique. En un sens large, toute forme de contrôle social qui barre une aspiration, impose des opinions ou des comportements, perturbe une trajectoire sociale ou un cadre de vie est violence, qu'elle soit ressentie douloureusement ou non par le sujet. On pense bien sûr, ici, au concept de "violence symbolique" chez Pierre Bourdieu'. Mais ce que Joan Galtung a appelé la "violence structurelle", par opposition à la violence personnelle et directe, illustre une conception encore plus extensive. Elle la définit en effet comme la pression sur les individus qui produit "une différence négative entre leurs possibilités d'accomplissement et leurs réalisations réelles" . Sous le concept de violence on comprend ainsi tout travail de socialisation et d'acculturation; toute exploitation d'une situation de nécessité dans les rapports marchands ; toute manifestation de puissance technologique qui remodèle brutalement l'espace où les hommes habitent. Mais il y a encore davantage : dans les sociétés contemporaines, le contrôle social ne se situe pas seulement dans cet entrelac de barrières juridiques, d'injonctions culturelles, de nécessités économiques. Il résulte encore d'irrésistibles sollicitations qui, exacerbant des désirs artificiels et contradictoires, morcellent le sentiment d'identité personnelle ; ce que l'on pourrait appeler les "violences de la séduction". Assimiler à la violence proprement dite toute forme de contrôle social conduit à reconnaître au phénomène une ubiquité qui en interdit pratiquement l'analyse. Il n'est pas non plus possible de s'en tenir aux représentations subjectives que s'en font les agents sociaux. Comme le remarque encore Yves Michaud, l'apparition de la violence "tient plus à la dissolution des règles qui unifient le regard social qu'à la réalité qu'elle peut avoir". En d'autres termes, elle constitue dans le langage l'indice d'une rupture de consensus. C'est pourquoi la dénonciation de la violence est moins l'indice d'une violence effective (il en existe bien d'autres qui ne sont jamais nommées) que "la mise en question de règles intolérables", le rejet de comportements considérés comme inacceptables. Il existe donc une véritable "fonction performative du concept". Dans ces conditions, il est bien clair qu'on ne saurait construire une définition opératoire de la violence politique à partir des perceptions contradictoires ou conflictuelles qui s'imposent dans les débats. Ce qui, à ce niveau, retiendra l'attention ce sont surtout les usages politiques du terme dans les discours de stigmatisation de l'adversaire ou les appels à l'émotion publique. L'analyse de science politique se doit de proposer un concept qui satisfasse aux critères 
de cohérence intellectuelle et de pertinence heuristique, tout en demeurant, selon le conseil de Durkheim, en connexion minimale avec les perceptions du sens commun ${ }^{10}$. L'expression: violence politique, tire sa richesse sémantique mais aussi son intérêt scientifique, du fait qu'elle éveille deux séries de connotations. Elle contraint d'abord à penser le problème de la place (résiduelle ?) occupée dans les systèmes démocratiques européens par l'emploi ou la menace de la force ; question d'autant plus importante que l'Etat de Droit peut sembler caractérisé par la forclusion de toute violence physique. L'expression véhicule également une charge émotionnelle qui oblige à porter attention à des dimensions psychosociales de la vie politique souvent négligées, et ce au prix d'une déperdition importante de ce qui fait sens pour les acteurs. L'emploi de la force, ou la menace d'y recourir, pose en termes particulièrement cruciaux le problème de la maîtrise des comportements d'agressivité ou de peur. Or la socialisation politique de type démocratique vise précisément, en Occident, à la mise en place d'une "autocontrainte" dont Norbert Elias nous dit qu'elle est "une régulation plus précise des manifestations pulsionnelles et émotionnelles selon un schéma différencié tenant compte de la situation sociale"11. Les grandes études empiriques ou historiques, conduites depuis trente ans $\mathrm{s}^{12}$, retiennent pratiquement toujours le critère de la violence physique, "usage délibéré de la force pour blesser ou détruire physiquement" (Gurr). S'il y a également large consensus pour distinguer les atteintes aux personnes et les dommages aux biens, les critiques en revanche ont été nombreuses contre la tendance de beaucoup de ces travaux à circonscrire les phénomènes de violence politique aux actions dirigées contre l'Etat ${ }^{13}$. Les distinctions violence/coercition, ou encore violence/force, qui mobilisent deux lexiques, l'un dramatisant, l'autre euphémisant, permettent de creuser un fossé de légitimité entre l'usage institutionnalisé de la contrainte matérielle au service de l'ordre politique et les usages protestataires ou contestataires. Cela encourage clairement des biais idéologiques au détriment des exigences de neutralité axiologique qui doivent soutenir la recherche. Ainsi très logiquement, dans une sorte de raisonnement symétrique de celui des défenseurs de l'ordre social, Georges Sorel appuyait-il sa justification de la violence révolutionnaire sur un distinguo analogue: "Nous dirons donc que la force (qu'il condamne) a pour objet d'imposer l'organisation d'un certain ordre social dans lequel une minorité gouverne tandis que la violence tend à la destruction de cet ordre"14. En réintégrant sous le concept unifié de violence politique des comportements qui ont tous en commun le recours à la contrainte matérielle, et cela indépendamment des systèmes de légitimation qui les "requalifient", on se donne les moyens de faire émerger de nouveaux questionnements sur le fonctionnement politique. C'est pourquoi on retiendra comme définition opératoire celle que proposait Nieburg, qui inspire d'ailleurs nombre de travaux, à savoir: "Des actes de désorganisation, destruction, blessures, dont l'objet, le choix des cibles ou des victimes, les circonstances, l'exécution, et/ou les effets acquièrent une signification politique, c'est à dire tendent à modifier le comportement d'autrui dans une situation de marchandage qui a des conséquences sur le système social" ${ }^{15}$. On le voit, cette démarche combine un critère purement matériel à savoir tout acte de force, indépendamment du statut de son auteur, et un critère purement politique : la signification acquise de l'acte. Sur cette base, il est possible d'identifier concrètement trois sous-ensembles. La violence d'Etat: la monopolisation tendancielle de la force physique est le fondement ultime de l'autorité du pouvoir politique: à l'extérieur dans le concert des nations, comme à l'intérieur où elle constitue la garantie d'effectivité de la règle de droit. Celle-ci, en effet, est une norme 
dont l'inexécution est sanctionnée par une peine (privative de biens, de liberté); sa mise en oeuvre exige le recours, ou la plausibilité du recours, à la contrainte contre les récalcitrants. Dans un Etat de Droit, l'emploi de la violence d'Etat, soigneusement codifié, bénéficie d'une présomption de légalité, même si sa légitimité suscite nécessairement des contestations, ne seraient-ce que réduites au groupe-cible. Cependant se développent parfois, y compris dans les démocraties occidentales, des pratiques parfaitement illégales. Les unes sont dues à des défaillances du contrôle institutionnel opéré sur les personnels d'exécution : bavures policières, autonomisation de facto de certains services, voire tortures ${ }^{16}$. Les autres sont imputables à l'initiative même des Pouvoirs Publics, notamment dans certaines conjonctures politiques (par exemple en relation avec la guerre d'Algérie et le terrorisme O.A.S., les activités des "Barbouzes"; ou encore les attentats d'intimidation contre des organisations contestataires, violentes ou non: I.R.A, E.T.A, Greenpeace). La violence protestataire : dirigée contre l'ordre social, le régime politique ou, simplement, les représentants et agents de la puissance publique, elle est susceptible de revêtir des modalités extrêmement variées. Violences armées d'organisations clandestines (I.R.A, E.T.A, F.L.N.C. ou, il y a quelques années, Fraction armée rouge, Brigades rouges...) ; violencesdéprédations d'émeutiers, de manifestants contre des immeubles publics ou des propriétés privées; agressions physiques ou menaces verbales contre des agents de l'Etat, des élus, des dirigeants politiques; emploi de la force matérielle, y compris la capacité d'obstruction du nombre, pour entraver le fonctionnement régulier des services publics : barrages routiers, sit-ins sur la voie publique, occupations sans titre de lieux privés ou publics, etc...La frontière peut d'ailleurs être très ténue entre le cortège autorisé qui provoque une gêne prévue et gérée par les Pouvoirs Publics, et celui, tout aussi légal à l'origine, qui suscite une désorganisation concrète. On observera que la violence comprise en ce sens inclut des formes d'action considérées parfois par leurs auteurs comme non violentes. En outre le consentement tacite de l'Etat n'efface pas nécessairement le caractère intrinsèque de l'opération menée. La violence intersociale : on entend par là les affrontements ou les menaces d'affrontements, qui mettent aux prises entre eux des groupes sociaux. Alain Corbin a montré combien, au $\mathrm{XIX}^{\circ}$ siècle, subsistaient de fortes traditions belliqueuses entre les villages ruraux ${ }^{17}$. De même les moeurs des compagnonnages étaient-elles d'une particulière brutalité à l'égard des apprentis; sous une forme à peine atténuée elle a longtemps perduré dans les usines. Evoquons enfin les antagonismes liés aux combats de la laïcité ou à l'intolérance religieuse. Mais aujourd'hui les facteurs les plus persistants de cette violence collective potentielle se situent ailleurs. Les crispations identitaires, liées à l'émergence générale des nationalismes en Europe, à partir du dernier tiers du XIX ${ }^{\circ}$ siècle, ont favorisé de nombreux phénomènes de rejet hétérophobique et, tout particulièrement, une relance de l'antisémitisme. Parallèlement l'essor de l'immigration étrangère, au XXe siècle, qui, avec des décalages chronologiques, concerne tous les pays de la zone sauf la péninsule ibérique et l'Italie, fait naître des problèmes plus ou moins aigus d'acceptation sociale. C'est notamment la question du racisme $^{18}$. La violence intersociale n'est pas intrinsèquement politique mais elle le devient en raison des effets qu'elle engendre éventuellement : ce sont essentiellement les possibilités stratégiques d'exploitation qu'elle ouvre au profit d'acteurs politiques. Dans les démocraties européennes contemporaines, le critère le plus clair du passage au politique est la requalification des faits en ce sens dans le débat public: médias, enceintes parlementaires, instances politiques. Cela ne s'opère pas toujours 
spontanément mais résulte parfois, au contraire, d'un travail acharné pour faire reconnaître le caractère politique des violences. Des observations fines permettraient d'identifier les codes qui sous-tendent ces processus et confèrent une probabilité plus ou moins grande de réussite aux efforts entrepris (suffit-il par exemple qu'il y ait implication directe de militants comme ce fut le cas lors des affrontements entre écologistes et aménageurs dans la Vallée pyrénéenne de l'Aspe en juillet 1992 ?). Trois dimensions du problème contemporain de la violence ont été retenues, non sans arbitraire peut-être. Leur dénominateur commun est de focaliser l'attention sur les fonctions psychosociales du recours à la force dans un Etat de Droit afin de mieux en comprendre la persistance et, peut-être, en gérer mieux les manifestations. La violence physique est d'abord un mode d'affirmation politique, qui a encore beaucoup d'avenir sous des formes probablement inédites. C'est aussi un comportement à (forte) charge émotionnelle, éventuellement générateur de séquelles traumatiques durables, dont la gestion par le système politique peut se révéler particulièrement délicate. C'est enfin à sa marginalisation maximale que tendent les diverses formes de contrôle social ; mais peut-on aller jusqu'au terme ultime de ce processus sans coûts excessifs pour le système politique lui-même?

\section{LA VIOLENCE COMME MODE D'AFFIRMATION POLITIQUE}

Les études empiriques, à caractère psychologique ou sociologique, mettent clairement en évidence ce processus : qu'il concerne les acteurs individuels eux-mêmes ou, à travers eux, une organisation sociale et notamment l'Etat. Cette affirmation de soi se prolonge politiquement dans un résultat. Intentionnellement ou ex-post, la violence s'inscrit dans un rapport de forces qu'elle influence, infléchit, voire recompose.

5 Construction d'une identité et exhibition de puissance C'est en effet dans cette double direction que se déploie l'affirmation politique par la violence. Dans l'action, ou la perspective de l'action, la dimension physique de l'affrontement catalyse de fortes solidarités; elle réintensifie le sentiment d'appartenance au groupe. J.K. Galbraith le notait à propos du lien entre l'affirmation de l'Etat et sa capacité de préparer (ou faire) la guerre. "La guerre, en tant que système social, écrivait-il, a non seulement constitué un élément essentiel de l'existence des nations en tant qu'entités politiques indépendantes, mais elle a également été indispensable à la stabilité intérieure de leurs structures politiques. Sans elle, aucun gouvernement n'a jamais été capable de faire reconnaître sa légitimité, ou son droit à diriger la société" ${ }^{19}$. Cette existence d'une menace, réelle ou supposée, constitue l'incitation la plus forte à imposer aux citoyens la conscience d'une allégeance nationale qui doit l'emporter sur toute autre et légitime, le cas échéant, le sacrifice de vies humaines. Elargissant son propos, Galbraith ajoute que "dans la vie quotidienne, cette situation est représentée par l'institution de la police, organisme armé chargé expressément de lutter contre les ennemis de l'intérieur avec des procédés militaires" ${ }^{20}$. La dimension: affirmation d'une solidarité, se décèle notamment chez les individus enrôlés dans une structure organisée : aussi bien du côté des militaires (la fameuse "fraternité de combat") et des forces de l'ordre ${ }^{21}$ que des militants de groupes clandestins en lutte contre le pouvoir établi. Mais sous une forme plus éphémère, elle est présente aussi au coeur de l'émeute (solidarités spontanées contre les "flics"). Plus troubles et plus illégitimes, celles-ci peuvent donner lieu après 
coup à des désolidarisations compensatoires enregistrées par exemple devant les micros des journalistes: "Nous ne sommes pas des casseurs..."(Los Angeles, printemps 1992) “Nous ne sommes pas des fascistes..." (Rostock, été 1992). C'est une caractéristique majeure du passage à la violence physique, toujours "simplificatrice", que d'exacerber la coupure entre amis et adversaires ${ }^{22}$, entre l'in-group et l'out-group. Et l'on sait, dans une guerre civile, le caractère particulièrement redoutable de la sommation à devoir "choisir son camp" ${ }^{23}$. Cette donnée, présente au coeur de toute violence collective, explique l'attrait qu'elle peut exercer sur certains styles de personnalités ${ }^{24}$. De même contribue-t-elle à éclairer certains modes d'engagement sur des bases autres que politiques ou idéologiques, dans l'armée et la police ou dans les mouvements de résistance, les milices armées, etc... ${ }^{25}$. Exprimer une identité par la violence c'est aussi, inévitablement, faire surgir un lexique autour duquel sympathisants et adversaires vont s'affronter pour tenter d'imposer une dénomination légitime: (Jeunes) Travailleurs en colère $\mathrm{v} / \mathrm{s}$ pègre ou populace, résistants $\mathrm{v} / \mathrm{s}$ terroristes, forces de l'ordre $\mathrm{v} / \mathrm{s}$ forces répressives ou flics. Les flambées de violences déprédatrices suscitent un travail d'identification et de qualification presque uniquement externe, les acteurs eux-mêmes s'évanouissant le plus souvent dans un silence que les enquêtes à chaud des médias tentent de rompre, avec un bonheur inégal. La bataille pour imposer les qualifications légitimes des événements et des acteurs est, par excellence, une bataille politique ${ }^{26}$. L'affirmation politique par la violence est encore et surtout une exhibition. Puissance physique, et impuissance politique tout à la fois. Lorsque Alexandre le Grand, au dire de Plutarque ${ }^{27}$, tranche l'inextricable noeud gordien d'un coup d'épée, c'est parce qu'il trouve plus rapide et plus efficace d'écarter spectaculairement les règles du jeu prévues par l'Oracle. Utiliser la violence dans les sociétés démocratiques où les processus d'institutionnalisation des conflits sont très développés, témoigne d'une impatience ou/et d'une impuissance à utiliser les procédures routinières de négociation et de représentation. Tant les observations empiriques que les justifications avancées par les acteurs, montrent que les recours à la violence sont basés sur la conviction qu'il n'existe pas réellement d'autre moyen d'obtenir la prise en considération de ses attentes ou, plus radicalement, d'imposer ses solutions. Même l'emploi de la force publique signale, comme le notait $\mathrm{H}$. Arendt, un échec du pouvoir politique à fonctionner dans son cadre normatif ${ }^{28}$. Les nombreux travaux analysés par E. Zimmermann ${ }^{29}$ mettent l'accent sur le sentiment de discrimination subie par le groupe contestataire, ou encore sur la perte de confiance dans le système politique. En ce sens, l'apparition de la violence est un "signal de danger" ${ }^{\prime 30}$ c'est-à-dire l'indice d'un dysfonctionnement du système politique : soit qu'il ait affiché une indifférence foncière au problème dont la violence est le révélateur, soit qu'il ait considéré les solutions préconisées comme radicalement inacceptables pour sa survie, soit enfin qu'il ait été incapable de percevoir correctement ou d'interpréter des signaux antérieurs de nature non violente. Notons, à propos de cette dernière éventualité, qu'il existe des violences, particulièrement des conflits armés, qui surviennent comme "effets émergents" d'une séquence de situations que personne n'a réellement maîtrisée, faute d'une lecture correcte des messages et réponses produits à chaque stade ${ }^{31}$. La violence comme mode d'affirmation d'un pouvoir de faire front, est une ressource politique inégalement disponible. S'agissant de la violence armée, l'Etat dispose d'une écrasante supériorité technique qui n'a cessé de s'accroître depuis l'époque où Engels, mais aussi Sorel, en tiraient quelques conclusions stratégiques essentielles $^{32}$. Mais encore faut-il que les conditions politiques de son emploi en soient 
réunies. Cependant l'évolution technologique a facilité aussi la banalisation d'armes redoutables, de même que l'apparition de cibles à haute dangerosité (centrale nucléaire, tunnel sous-marin, ouvrage d'art...). Plus que la capacité de vaincre, la capacité de nuire gravement est à la portée de groupes bien organisés, même petits, qui remplissent quelques conditions-clés: soutiens extérieurs, sympathies au sein de l'appareil répressif d'Etat, "professionnalisme". Quant à la violence non armée, (à mains nues ou avec de simples instruments de, ce que les juristes appellent des armes par destination) la plausibilité d'y recourir renvoie à d'autres critères de discrimination qui sont pour l'essentiel socioculturels. Il existe des groupes sociaux où l'exercice physique (les militaires, les professionnels du sport), le travail manuel et la force musculaire (les ouvriers, les paysans...) constituent encore un facteur important de l'adaptation professionnelle ${ }^{33}$. La valorisation de l'exploit physique semble y créer une propension plus importante à admettre, le cas échéant, la violence à mains nues. Ce sont aussi, pour certains d'entre eux, des groupes sociaux moins bien armés pour utiliser les ressources spécifiquement politiques du système institutionnel, ne serait-ce qu'en raison de leur sous-représentation dans les institutions publiques. C'est pourquoi la légitimation (relative) ou la stigmatisation absolue de la violence physique est sourdement un enjeu dans les antagonismes de classe ${ }^{34}$. Le "handicap culturel" de ce répertoire d'action s'ajoute à celui de son illégalité, sauf dans le cas de la violence d'Etat. D'où l'importance politique des systèmes de légitimation allégués: "la juste colère des travailleurs", "la répression policière", "le désespoir de la paysannerie" ou, naguère, "la Cause de la Révolution". Pour des raisons sociales et culturelles profondes, la dynamique de l'Occident marque d'un stigmate croissant ceux qui, dans le système démocratique, cherchent à s'imposer comme acteurs par la force. Ce qui néanmoins n'exclut pas, loin de là, l'existence du phénomène.

6 La violence dans les logiques de négociation et marchandage Anthony Oberschall observe que la violence est un moyen parmi d'autres de conduire un conflit et que, souvent, elle est associée étroitement à des moyens pacifiques employés concurremment ${ }^{35}$. Il en résulte d'importantes conséquences. Dans le souci de préserver les chances d'un dialogue que l'on sait devoir accepter, il est fréquent de voir les protagonistes modérer l'ampleur du recours à la force. En revanche, une violence soft parait à de nombreux contestataires comme le moyen efficace de s'imposer à la table de négociation dans un système démocratique; du moins à deux conditions. La première est l'exigence de visibilité: il faut que les médias en parlent. La couverture journalistique joue un rôle décisif d'orchestration ou d'amplification ${ }^{36}$. Mais leur logique est de privilégier en ce domaine le spectaculaire ou l'inédit qui frappe l'imagination. La seconde condition est de ne pas franchir un certain seuil au delà duquel elle deviendrait contre-productive dans la perspective de négociation. Soit parce qu'elle provoquerait le surgissement de "préalables"; soit parce qu'elle déclencherait l'apparition de réflexes sécuritaires susceptibles de faire passer au second plan les problèmes de fond qu'on avait voulu imposer sur le devant de la scène ; soit enfin parce qu'elle risquerait de légitimer en réponse un recours à la force susceptible d'enclencher un scénario d'escalade plus difficilement maîtrisable. Ainsi existe-t-il dans nos sociétés des formes de violence modérées, banales, acceptées. Inscrites de facto dans le jeu institutionnel routinier, ces formes de violence (occupations illégales de la voie publique, barrages routiers, déprédations relativement bénignes, actions plus spectaculaires que brutales, séquestrations à la fois brèves et sans brimades notables) constituent, dans l'intervalle des consultations électorales, une 
manière de "faire de la politique d'une autre façon" ${ }^{37}$. Le plus singulier sans doute est le fait que l'Etat démocratique lui-même est conduit non seulement à tempérer l'emploi des forces de l'ordre mais à tolérer délibérément un niveau de violences modérées et momentanées, parfois en parfaite connivence avec l'“adversaire" ${ }^{38}$. Cet Etat ne pratique plus, comme avant le $\mathrm{XIX}^{\circ}$ siècle ou dans les dictatures contemporaines, une stratégie de "représailles massives" mais une "riposte graduée" qui s'inscrit dans une logique de compatibilité avec l'exigence de libre expression. La culture démocratique tolère des marges de violence non seulement verbale mais également physique sans s'en trouver déstabilisée. Cependant il est une importante exception à cette réalité d'une intégration de facto de la violence dans le jeu politique routinier. C'est le cas où la violence exercée s'inscrit dans une logique de terrorisation. La stratégie de la terreur est un paroxysme de l'affirmation de soi, intégralement négateur de l'existence d'autrui. D'où son rapport particulièrement étroit avec la mort infligée. Elle ne relève plus, intrinsèquement, d'une recherche du compromis, du dialogue, de l'échange; elle cherche à paralyser. Paralyser les résistances de la Société ou paralyser la volonté de l'Etat, cherchant à mettre en place une sorte de rapport éthologique de proie à prédateur. Comme l'écrit D. Apter: "La cible commune du terrorisme est la légitimité de l'Etat, sa crédibilité morale...Le premier acte du drame social du terrorisme consiste en une bataille du bien contre le mal, la plus ancienne de toutes les histoires de l'humanité. Mais le deuxième acte tend à créer un moment disjonctif, point de rencontre des crises" ${ }^{\prime 3}$. Le critère de l'entreprise de terrorisation est le franchissement délibéré de tous les "seuils d'acceptabilité sociale". Rien sans doute n'est plus subjectif que cette notion de seuil dont on saisit tout de suite la relativité selon les situations vécues par les agents sociaux. Pourtant on repère clairement les traces d'un travail à la fois policier, politique et médiatique tendant à identifier des paliers de violence en termes de défis à gérer, de réponses politiques à apporter, de couverture journalistique à assurer. De même, l'expérience de S. Milgram avait elle permis de tester des seuils de résistance au commandement de "l'autorité légitime" ordonnant d'infliger des souffrances ${ }^{40}$. La recherche de l'intensité du stress qui est au coeur de la stratégie de terreur, implique d'abord le franchissement de la barrière du sang versé. Entre les dommages causés aux biens et les blessures délibérément infligées aux personnes il existe un fossé dont rend compte un traitement médiatique en général extrêmement différent ${ }^{41}$. Un second type de seuil se situe sur le terrain du choix des cibles. La stratégie de terrorisation cherche à briser progressivement le sentiment de sécurité en écartant toutes barrières qui sembleraient mettre à l'abri telles ou telles catégories de populations; d'où en retour ces expressions: "attentats aveugles...victimes innocentes.." ${ }^{42}$. Un troisième type de seuil renvoie à une sorte d'escalade de l'intensité : nombre des victimes, fréquence des actions, renchérissement des exigences... Sous le regard attentif des media, aujourd'hui : "Terrorists choreograph their violence"43. Avec des moyens incomparablement plus puissants le terrorisme d'Etat obéit, lui aussi, à cette logique d'insécurisation généralisée. Les régimes nazi et stalinien avaient aboli toute stabilité de la loi et toute définition restrictive des infractions ${ }^{44}$. Tendanciellement, personne ne pouvait se considérer comme totalement à l'abri de leur appareil répressif. Et cette logique de destruction impliquait aussi bien la dégradation psychologique que l'élimination physique des "adversaires". Les stratégies de terrorisation à large échelle entraînent nécessairement des séquelles traumatiques. C'est là d'ailleurs que se joue une différence essentielle entre la violence physique et des "violences" qui demeurent purement économiques ou symboliques. Transmise d'une génération à l'autre, la 
mémoire de l'horreur constitue une donnée essentielle pour la compréhension d'un comportement contemporain comme l'expression d'identité juive dans les démocraties occidentales. Sur un tout autre plan la notion de séquelle traumatique permet également de mieux appréhender la manière dont se construisent certains comportements fondés sur la culpabilité ${ }^{45}$ ou sur le déni (les "blancs de l'histoire" repérables en Allemagne mais aussi en France ou en Espagne).

\section{LA DUALITE : VIOLENCE COLERIQUE/VIOLENCE INSTRUMENTALE}

L'implication émotionnelle des acteurs dans la violence qu'ils mettent en oeuvre est une donnée importante à prendre en considération car elle appelle des modes de gestion politiques qui ne sont pas identiques. C'est l'intérêt de distinguer une violence colérique, souvent mais pas toujours, liée à des pratiques protestataires ${ }^{46}$, et une violence instrumentale calculée, graduée, qui est en principe le mode normal d'intervention de l'Etat démocratique à l'intérieur comme à l'extérieur. La première est en quête de profits psychologiques immédiats, au niveau d'une libération de tensions insupportables, la seconde se situe dans un rapport très politique : fins-moyens. Il s'agit cependant de deux modalités ideal-typiques, c'est-à-dire qu'elles servent davantage comme guide de lecture des réalités observables que comme description empirique des faits. Elles se rencontrent en effet rarement à l'état pur. Il convient donc d'utiliser cette distinction comme moyen de déchiffrer l'inégale présence de cette double dimension dans les comportements effectifs.

La violence colérique Elle peut être définie comme un acting out destructeur provoqué par une décharge d'agressivité. C'est donc le lien qu'elle entretient avec cette "disposition" psychologique qui lui confère son dynamisme propre, notamment dans son mode de surgissement et d'épuisement ${ }^{47}$. Si on laisse de côté les thèses éthologistes sur l'agressivité qui soulèvent la question - insoluble en sciences sociales - de son caractère inné ou acquis, deux grandes problématiques psychosociologiques fournissent des clés pour en comprendre le fonctionnement politique. Ce sont d'abord les théories de la frustration relative auxquelles les travaux de Gurr et des Feierabend ont fourni un ample matériau empirique. Emprunté par eux à Berkowitz le concept de frustration peut se définir comme la différence entre un plaisir escompté comme accessible ou légitime, et la réalité d'une privation ${ }^{48}$. Selon Berkowitz, la frustration engendre la séquence : colère... identification d'une cible... infliction d'un dommage... diminution de la pulsion agressive par assouvissement partiel...réduction (momentanée) du sentiment de frustration. Cependant il ne s'agit que d'un scénario de réponse possible. Divers facteurs, notamment psychologiques, socioculturels et politiques, peuvent favoriser d'autres séquences : blocage du passage à l'acte avec, par exemple, retournement contre soi de l'agressivité, ou encore résignation dans le ressentiment. Les enquêtes précitées concluent que les frustrations d'ordre économique dans une population hétérogène sont les plus porteuses de violences, bien davantage que les frustrations engendrées par les inégalités de statuts, de participation, ou encore de perspectives d'autoréalisation ${ }^{49}$. Mais, bien entendu, ces diverses sources de frustrations peuvent se cumuler, donc augmenter la plausibilité du passage à la violence (catholiques de l'Ulster). A noter que cette analyse probablement adaptée à l'interprétation des violences sociales aux Etats-Unis ne saurait rendre 
compte sans difficultés de l'absence de violence politique dans les communautés de travailleurs immigrés en Europe ${ }^{50}$. L'autre grande catégorie d'interprétations du lien entre agressivité et violence met l'accent sur les mécanismes culturels d'apprentissage et de valorisation des comportements agressifs. Dans son ouvrage de référence Albert Bandura minimise l'importance initiale des phénomènes purement émotionnels comme le ressentiment, la fureur, la colère. "Une culture, écrit-il, peut produire des gens hautement agressifs tout en maintenant un faible niveau de frustration, en valorisant les performances agressives, en fournissant des modèles d'achèvement de type agressif, en assurant à ceux qui recourent à des actions agressives des gratifications ou des récompenses" ${ }^{\prime 1}$. Au sein des sociétés occidentales, il est intéressant de repérer des subcultures, propres à des classes sociales ou à des groupes sociaux, qui se révèlent plus accueillantes à l'affirmation de soi dans la violence physique, cultures ayant conservé davantage d'inclination pour les "valeurs viriles" ${ }^{2}$. Les caractéristiques essentielles de la violence colérique peuvent être rassemblées autour de deux éléments : . Le premier est la suspension, au moins partielle au coeur de l'action, du calcul rationnel : coûts/ avantages. Ira brevis furor. (Sénèque) L'intensité de la violence exercée peut en effet être tout à fait contre-productive politiquement; le choix des cibles apparemment absurde (par exemple l'attaque de perceptions pour protester contre la pression fiscale). Cela ne signifie pas qu'elle ne puisse être instrumentalisée c'est à dire intégrée ex-post comme élément d'un calcul politique, voire délibérément provoquée dans ce but. Il y a en effet manipulation inévitable de la colère par des agents politiques qui vont l'exploiter, l'interpréter, bref tenter d'en tirer un profit politique. Mais par nature, elle ne se laisse pas enfermer dans des limites rigoureusement prévisibles; elle n'est donc pas facile à réguler par ceux qui l'instrumentalisent ${ }^{53} \mathrm{ou}$ la redoutent. Il est difficile à quiconque, y compris aux acteurs eux-mêmes (on pense notamment aux phénomènes de foule) d'anticiper avec certitude le moment, les formes et l'intensité de ces phénomènes souvent qualifiés métaphoriquement de volcaniques. On l'observe aisément avec les récentes émeutes urbaines, en France, en Grande-Bretagne, en Allemagne, qui ont pris à contre-pied gouvernants et observateurs. Il s'ensuit, et ceci est politiquement très important, une finalisation approximative de l'acting out. Avant tout libération de tensions agressives accumulées, la violence colérique des acteurs ne se donne pas d'emblée des objectifs politiques très élaborés ni même parfois totalement cohérents. Fréquemment, le mouvement éclate avant que n'ait été définie une plateforme revendicative. Celle-ci se trouve rédigée, ou du moins complétée, après coup ; et pas toujours par les auteurs eux-mêmes des violences. Elle le sera lorsque doivent s'ouvrir des négociations ou, tout simplement sous l'injonction des questions posées par les journalistes: "Mais quels sont donc les objectifs de votre mouvement...?". En revanche, il est fréquent qu'au coeur de l'action s'exprime fortement une dimension véritablement ludique, que $M$. Maffesoli évoque lorsqu'il propose l'expression de violence orgiaque ${ }^{54}$. L'ivresse de "tout casser" ou de "cogner les flics" se situe dans l'univers de la gratuité politique même si, bien entendu, d'autres acteurs s'apprêtent à l'intégrer dans leur calcul pour susciter la peur par exemple, ou au contraire faire sortir des réformes de l'ornière. Cette dimension rend différente la gestion du phénomène de violence dans le cadre du système politique démocratique. . Le second est l'identification d'une victime émissaire. Cette notion doit être soigneusement distinguée de la simple cible d'une violence calculée. Comme l'écrit René Girard, "la violence inassouvie cherche et finit toujours par trouver une victime de rechange. A la créature qui excitait sa fureur, elle en substitue une autre qui n'a aucun titre particulier 
à s'attirer les foudres du violent, sinon qu'elle passe à sa portée"55. La thèse centrale de Girard est qu'un tel mécanisme fonde l'institution des rites sacrificiels. A la violence de tous contre tous et à son cycle répétitif qui menace le groupe et ses membres, va se substituer un événement symbolique résolutoire: jadis le sacrifice religieux; aujourd'hui le travail juridique du juge ou encore, ajoutera-t-on, les diverses procédures de responsabilité politique. Mais l'explosion de violence colérique est précisément une mise en échec, au moins momentanée, de ces mécanismes de contrôle social. Il y aura donc régression du rituel, à forte capacité symbolique, vers des pratiques de pure violence physique. "Toute communauté en proie à la violence ou accablée par quelque désastre auquel elle est incapable de remédier se jette volontiers dans une chasse aveugle au bouc émissaire. Instinctivement, on cherche un remède immédiat et violent à la violence insupportable. Les hommes veulent se convaincre que leurs maux relèvent d'un responsable unique dont il sera facile de se débarrasser. On songe tout de suite, ici, aux formes de violences collectives qui se déchaînent spontanément dans les communautés en crise, aux phénomènes du genre lynchage, pogrom, justice expéditive, etc..." ${ }^{56}$. La désignation du bouc émissaire obéit à une logique spécifique: non pas une victime coupable mais une victime sacrifiable. Les victimes ne sont pas choisies en raison des "crimes" qu'on leur attribue mais des "signes victimaires" qui les caractérisent. Elles sont à la fois "différentes" de l'in-group, mais présentes et visibles à ses yeux; elles appartiennent par exemple à une minorité différenciée ou encore se situent dans le groupe mais à la marge, soit aux sommets de la hiérarchie sociale (les "grands", le Prince...) soit à sa périphérie dépréciée. "Lorsque un groupe humain, ajoute Girard, a pris l'habitude de choisir ses victimes dans une certaine catégorie sociale, ethnique, religieuse, il tend à lui attribuer les infirmités ou les difformités qui renforceraient la polarisation victimaire si elles étaient réelles" ${ }_{57}$ (caricatures racistes par exemple mais aussi législations discriminatoires). Comment gérer cette forme spécifique de violence dans un système démocratique? Les réponses envisageables sont susceptibles de se situer théoriquement à deux niveaux. Le premier est celui du traitement des situations concrètes génératrices de cette irruption de colère. En réalité de nombreux obstacles entraveront une action efficace. Il n'est pas simple de s'attaquer effectivement aux inégalités économiques et culturelles tout en évitant d'aggraver les tensions sociales; encore moins facile d'obtenir des résultats rapides en s'en prenant aux schèmes culturels qui valorisent les comportements violents. Il n'est même pas sûr qu'une telle démarche (la première surtout) soit toujours pertinente dans ce but. Le second niveau d'intervention, mieux adapté au moins à court terme, est celui d'un travail plus proprement symbolique ${ }^{58}$. La volonté d'affirmation, voire la revendication confuse de participation politique qu'exprime le recours à la violence, appelle en retour des "gestes", au sens tocquevillien du terme, de prise en considération : ouverture d'un dialogue, même et surtout s'il est rugueux; stigmatisation moins des personnes que des actes de violence inacceptables; travail psychologique et politique de dissociation/discrimination entre les comportements condamnés. L'écueil principal est alors d'éviter de franchir le seuil au-delà duquel la violence apparaît "récompensée" par des gratifications, donc encouragée à éclater de nouveau. Par ailleurs, les mécanismes de responsabilité politique en démocratie offrent, mieux que dans d'autres régimes, une issue à la quête de victimes émissaires acceptables. Ce sont les fameux "fusibles" qui permettent de sacrifier des "responsables", contribuant ainsi à exorciser les sentiments d'impatience et 
d'impuissance qui se situent à l'origine de la violence. "Quelque chose se passe.. !" Ainsi s'opère un travail de resymbolisation qui est de l'essence même du politique.

10 La violence instrumentale Elle est exercée, sans passion ni agressivité incontrôlée, en vue d'atteindre des objectifs définis. Elle s'inscrit directement dans une logique de calcul et d'efficacité ; ce qui implique la recherche consciente d'une proportionnalité des moyens mis en oeuvre par rapport au but recherché. Cette modalité de la violence est censée caractériser, tout d'abord, la coercition d'Etat. L'ambition des dirigeants démocratiques, les attentes générales de la Société vont dans le sens de cette discipline rigoureuse des personnels spécialisés dans la mise en oeuvre éventuelle de la force. Il est loin le temps où les chefs de guerre devaient concéder à leurs troupes le sac des villes prises même si subsistent aujourd'hui des traces de cet état d'esprit dans certaines conjonctures (difficultés à faire traduire devant les tribunaux les auteurs d'excès). Lorsque, face à la contestation intérieure, l'Etat déploie son appareil de contrainte, la préoccupation d'éviter les "bavures policières" est devenue, chez les gouvernants, un objectif de premier plan en raison des conséquences politiques négatives susceptibles d'en surgir. Vieille exigence de la démocratie libérale, l'emploi de la force doit être proportionné à la gravité du trouble à l'ordre public. On observe, en longue période, comme le montrent bien les travaux en cours de Patrick Bruneteaux, un double mouvement tout à fait caractéristique de ce point de vue. D'une part une professionnalisation accrue des personnels déployés; d'autre part un renforcement de leur formation technique et psychologique qui accorde une place centrale au contrôle de l'émotivité. Cependant la violence instrumentale n'est pas l'apanage du seul Pouvoir d'Etat dans les démocraties européennes contemporaines; elle est aussi un mode d'expression contestataire. Le recours à une violence calculée et calculatrice est le fait d'organisations, extérieures mais aussi indigènes (du type I.R.A. , F.L.N.C. corse, E.T.A. basque...), dont c'est le mode "normal" d'insertion dans le jeu politique, compte tenu de leurs objectifs et des moyens réels dont elles disposent pour maximiser leur affirmation politique. Bien entendu, ces organisations recrutent en partie sur la base de ressentiments actifs. Mais elles n'atteignent leur pleine efficacité qu'au prix d'une discipline impitoyable et d'une professionnalisation certaine. Souvent le militant convaincu y côtoie le "technicien" fasciné par les armes, voire le mercenaire motivé par l'argent ${ }^{59}$. Par ailleurs des organisations parfaitement légales (syndicats, groupes de pression, partis) peuvent également recourir délibérément à des formes soft de violence. Il s'agit d'affirmer une représentativité sur le terrain en démontrant une capacité d'entraver et, plus largement, de mobiliser. Selon la thèse développée par Charles Tilly, la violence est envisagée, par les challengers des détenteurs du pouvoir, comme une ressource parmi d'autres (le répertoire d'action) leur permettant de s'imposer dans le jeu politique. Si les gains escomptables sont élevés et les coûts réduits, notamment en termes de répression, alors la probabilité d'un recours à la violence augmente dès lors que surgit une conjoncture favorable, c'est-à-dire une mobilisation croissante autour des revendications rejetées par les gouvernants ${ }^{60}$. La violence est donc le résultat d'un processus. Cependant les partis, plus que les syndicats ou les groupes d'intérêts, doivent être particulièrement attentifs à l'éventuelle contreproductivité des interventions de ce type du fait que leur répertoire d'action, en démocratie pluraliste, est principalement orienté vers les luttes électorales. Une caractéristique majeure de cette forme de violence, d'ailleurs également soulignée par Charles Tilly, est l'exigence d'organisation, voire d'institutionnalisation. Il est impératif, en effet, de conserver de bout en bout la totale maitrise de l'action, malgré 
les risques permanents de dérapages. Normalement l'Etat est assez bien placé pour satisfaire à cette condition, mais aussi des organisations fortement structurées (en témoigne l'organisation toute militaire du syndicalisme paysan en Bretagne dans les années soixante, ou encore celle du service d'ordre C.G.T.) ${ }^{61}$. Ainsi peut-on avancer que l'affirmation croissante d'une violence purement instrumentale dans les sociétés européennes occidentales est corrélée avec ces processus d'institutionnalisation qui les travaillent en profondeur depuis plusieurs siècles. Elle contraste avec l'extrême cruauté qui accompagnait encore massivement, au XVIII ${ }^{\circ}$ siècle, la violence d'Etat, les jacqueries paysannes, ou les journées révolutionnaires. Cela ne signifie pas que cette évolution limite nécessairement l'ampleur des destructions ou des crimes. Lorsque aucun frein ne restreint son emploi "rationalisé" au service de fins totalitaires, on se trouve confronté à une logique d'extermination programmée, méthodique et froide comme le fût la "solution finale" mise en oeuvre par le III ${ }^{\circ}$ Reich. A la différence de la violence colérique, la violence instrumentale appelle directement l'attention sur les objectifs qu'elle sert. C'est donc à ce niveau qu'elle doit être gérée. Encore faut-il que les exigences formulées soient négociables dans le cadre du système politique. Dans l'affirmative, la conclusion d'un compromis acceptable par les deux parties entraîne une disparition effective de la violence. Mais le risque encouru est d'encourager d'autres acteurs à mobiliser à leur tour une ressource politique qui s'est révélée payante. Les répertoires d'action violente sont suffisamment diversifiés, la Société contemporaine suffisamment fragile avec ses mécanismes sophistiqués, pour que le recours à cette ressource politique apparaisse à beaucoup comme une véritable tentation. Il est très possible qu'à l'avenir se multiplient des actions contestataires violentes, à niveau d'ailleurs fréquemment modéré, surtout si devait s'approfondir la crise des institutions classiques de représentation, notamment au niveau européen où le déficit démocratique est le plus aigu.

\section{LA VIOLENCE ET LE RENFORCEMENT DU CONTROLE SOCIAL}

12 Le "processus de civilisation", pour parler comme Elias ${ }^{62}$, tend globalement à la dévalorisation en longue période du recours à la force. Mais il existe un coût social et politique de sa forclusion qui mérite d'être mis en évidence.

13 La marginalisation de la violence physique comme exigence de fonctionnement des sociétés contemporaines Témoin d'un basculement des valeurs qui s'opérait sous ses yeux, Spencer opposait sociétés militaires et sociétés industrielles. Les premières ne craignaient pas de légitimer hautement la nécessité de la violence comme moyen d'assurer le destin du groupe. Elles cultivaient l'éloge des vertus guerrières à travers les figures emblématiques du "soldat-paysan" attaché à sa terre (Rome), du "chevalier" épris de prouesses sur le champ de bataille comme dans les tournois (Moyen-Age), du condottiere ou du conquistador réalisant pleinement un idéal brutal d'affirmation héroïque (Renaissance). Et l'on sait la part du culte de la force dans les discours d'exaltation patriotique jusqu'au XXe siècle. La conquête de la gloire, valeur référentielle suprême dans ces sociétés, était indissociable des combats et périls physiques qu'ils faisaient encourir. Mais cet éloge de la guerre, si présent dans l'oeuvre d'un Joseph de Maistre ou même d'un Proudhon, ne se réduisait pas à des significations purement militaires. L'apologie de la violence révolutionnaire par Sorel était 
explicitement rapportée à ce qu'il appelait "la conception noble de la guerre"63. Quant à Lénine, stratège de la conquête du pouvoir, Perry Anderson a souligné combien il affectionnait les métaphores militaires qui abondent effectivement dans son oeuvre ${ }^{64}$. L'éloge de la violence, mise au service de la révolution, prolonge une attitude antérieure de célébration de la violence physique au service d'une juste cause : la gloire de Dieu dans la figure du croisé, le redressement des torts chez le bandit d'honneur (Hobsbawm), l'exigence de laver un affront dans le code d'honneur des gentilshommes mais aussi des bandes rivales de villages ou des gangs mafieux. Les sociétés contemporaines, parce qu'elles sont démocratiques mais aussi, et surtout peut-être, parce qu'elles sont marchandes, font totalement basculer les représentations de la violence physique. Non sans se trouver confrontées à quelques contradictions. . L'activité économique fortement intégrée exige une pacification particulièrement poussée des rapports sociaux. La violence n'est pas seulement destructrice (en quoi déjà elle entrave les processus d'accumulation du capital productif) ; elle est bien davantage désorganisatrice, heurtant alors de plein fouet les logiques de rationalisation du travail et d'interdépendance croissante des échanges qui caractérisent les systèmes économiques développés. Si le capitalisme est agressif, ce n'est pas d'abord, comme le pensait Lénine, en poussant à la guerre; c'est en jouant sans merci des lois de la concurrence. Or la "violence économique" peut n'être pas moins douloureuse que la violence physique ${ }^{65}$. On le voit bien en Allemagne aujourd'hui où la destruction du tissu économique hérité du régime socialiste de la R.D.A. crée, au moins transitoirement, des situations proches du désespoir. La dureté des logiques purement "économistes" engendre des facteurs théoriquement favorables à un retour de la violence. En effet la perte collective de l'emploi, la destruction d'un environnement familier par des "équipements lourds", la constante pression sur le consommateur sont à l'origine de frustrations nombreuses qui doivent être socialement gérées. Des législations par exemple visent à atténuer les rigueurs résultant d'une exclusive prise en compte des exigences économiques dans la gestion des entreprises : on pense bien sûr au régime de l'allocation-chômage ou aux transferts sociaux. Mais leur efficacité dépend en dernière analyse du maintien à bon niveau des performances économiques, qui ne saurait être indéfiniment garanti en longue période. En outre, il n'est nullement assuré que les conséquences de la liberté d'établissement, dans les pays de la CEE, seront toujours faciles à maîtriser; des difficultés économiques graves et persistantes peuvent faire naître des tensions entre communautés qui cohabiteront sur un même territoire. Une grande attention préventive devrait être apportée à cette éventualité. . Le triomphe contemporain des valeurs de la démocratie pluraliste en Europe entraîne la forclusion officielle de toute violence physique comme moyen de contester la loi ou d'obtenir la solution d'un conflit. La seule arme reconnue en pleine légitimité aux mécontents est celle du bulletin de vote. Elle l'emporte sur toute autre. Parallèlement, c'est le déploiement de la violence d'Etat qui s'entoure d'une circonspection croissante. L'idéal démocratique postule en effet que la loi s'impose normalement du fait de sa seule légitimité ; parce qu'elle est l'expression de la Volonté générale. Pourtant, il est non moins vrai que la force du droit, l'autorité de la loi reposent, en dernière instance, sur le monopole de la coercition, c'est à dire sur la violence. Ce qui caractérise l'Etat de droit, par opposition à tous les régimes tyranniques, ce n'est pas l'impossible renonciation à la force mais la soigneuse codification de ses conditions d'emploi. La norme juridique demeure une injonction ou un interdit dont le non-respect justifie, au terme de garanties procédurales, le recours à la contrainte matérielle. Malgré cette 
réalité incontournable, la formulation selon laquelle la démocratie repose sur la force matérielle, celle de la police et des prisons est intolérable. Intolérable aux gouvernants dont elle saperait indirectement l'autorité en facilitant la légitimation des contestations par la violence; intolérable aux gouvernés dont l'obéissance à la loi devient narcissiquement moins coûteuse si elle n'apparaît pas comme la soumission à la force. C'est pourquoi on observe la mise en place de mécanismes élaborés destinés à gérer cette contradiction: . discours d'occultation de la violence derrière une célébration appuyée de la souveraineté du Peuple , du "bon citoyen" qui respecte la Loi , paie ses impôts et se rend aux urnes pour exercer son droit de vote. . discours d'euphémisation qui introduisent les distinctions entre violence et coercition, violence et contrainte d'intérêt général. . discours de déni construits sur l'opposition entre solution politique d'un conflit et solution de force. En réalité la solution politique est toujours appuyée sur la construction d'une situation, en termes de légalité et légitimité, qui rend l'usage effectif de la force - mais non sa présence en arrière plan comme garantie d'effectivité - simplement inutile en raison de la disproportion créée entre les protagonistes ${ }^{66}$. Mais le discours démocratique est soumis à un défi spécifique lorsque le libre jeu du suffrage universel ouvre aux ennemis de la démocratie la plausibilité d'un accès au pouvoir d'Etat. Les événements de janvier 1992 en Algérie ont réactivé le débat sur l'acceptabilité d'un recours visible à la force pour annuler les résultats d'un scrutin. Nul doute qu'il gagnerait en intensité dramatique si un jour, dans un pays européen, se trouvaient réunies des conditions politiques analogues à celles que la popularité du F.I.S. avait créées sur l'autre rivage de la Méditerranée.

Le renforcement du contrôle social comme garantie de marginalisation de la violence politique Cette proposition peut sembler surprenante puisque les sociétés occidentales sont à la fois plus permissives et moins violentes depuis que la démocratie pluraliste s'y est consolidée dans les quatre dernières décennies. Pourtant de trois points de vue théoriques différents on se trouve orienté vers la même conclusion. . La problématique éthologiste qui rapporte la violence à l'agressivité, souligne que dans le règne animal, celle ci fait l'objet de blocages instinctuels spécifiques pour en interdire des usages dévastateurs, notamment au sein de l'espèce. L'Homme a perdu l'usage des automatismes inhibiteurs présents en amont de l'évolution alors qu'il est devenu capable d'une violence infiniment supérieure à celle du règne animal. En outre, ses conditions d'existence multiplient les occasions de déclenchements d'agressivité : densité de population, intrication des "territoires", langages complexes facilitant les mésinterprétations. Il faut donc qu'il existe de puissants relais pour suppléer les instincts défaillants; ce sont les contrôles culturels ${ }^{67}$. . La problématique de l'apprentissage (Bandura, Skinner) débouche sur l'idée que les modèles de comportements proposés à l'observation peuvent contribuer, s'ils sont non agressifs, au contrôle de la violence physique. On se trouve notamment confronté ici à la question de savoir si le spectacle de la violence politique dont la télévision se nourrit pour des raisons d'audimat, facilite l'imitation ou, au contraire, joue un rôle cathartique de purgation symbolique. Une énorme littérature s'est penchée sur cette question qui aboutit à des conclusions globalement indécises, ce qui s'explique aisément. En effet, il faut prendre en considération de très nombreuses variables : la coexistence de modèles agressifs et non agressifs, l'inégale valorisation qui en est proposée, l'inégale possibilité offerte aux téléspectateurs de s'identifier aux auteurs de violences compte tenu des prédispositions des premiers et du statut sociopolitique des seconds ${ }^{68}$. En cas de violence intense, le spectacle particulièrement coûteux psychologiquement de ses 
effets dramatiques, en termes de souffrances infligées à des innocents, réactive des inhibitions culturelles apprises ${ }^{69}$. Encore faut-il s'attendre à ce que ce mécanisme joue de façon sélective sur des personnes capables d'empathie. . La problématique psychosociologique explique le passage de la frustration à la violence en faisant intervenir trois catégories de variables ${ }^{70}$. Ce sont les justifications normatives ou éthiques de la violence, surtout si elles peuvent être rapportées à des autorités particulièrement légitimes; ce sont ensuite les justifications utilitaires c'est à dire la conviction que la violence paie parce que les "punitions" ne sont ni sévères ni certaines; ce sont enfin les aptitudes à la fois techniques et politiques du régime gouvernemental à combattre la violence (y compris la capacité d'obtenir des soutiens extérieurs à la répression ou de réduire ceux des contestataires). A ces trois niveaux ce sont bien diverses modalités de contrôle social qui peuvent jouer le rôle de frein efficace à l'apparition de la violence politique: le renforcement de légitimité des discours de stigmatisation et l'efficacité accrue dans la poursuite des auteurs de violences. Conformément à ces problématiques on observe effectivement des manifestations de l'accroissement du contrôle social au sein des démocraties européennes. Tout d'abord cela concerne leur propre appareil de contrainte qui est mieux tenu en mains politiquement, mieux formé professionnellement et psychologiquement ${ }^{71}$. Cela s'exprime aussi par une pression culturelle accrue pour disqualifier les recours à la force. Les justifications idéologiques dotées d'un minimum d'autorité intellectuelle ont quasiment disparu avec l'évolution du marxisme occidental et l'obsolescence des discours révolutionnaires ${ }^{72}$. Et si les médias montrent beaucoup la violence, cette présentation est rarement accompagnée d'un commentaire qui la place sous un jour favorable, à l'exception récente de la guerre du Golfe ${ }^{73}$. Même s'il est permis de s'en féliciter, cette évolution, assez récente (postérieure à la fin des guerres coloniales), assure la mise en place d'un contrôle social accru par disparition de discours dissonants. Ses effets sont peut-être visibles dans la mise en échec, au cours des décennies soixante-dix et quatre-vingts, des tentatives de déstabilisation en R.F.A. ou en Italie, due largement à la désolidarisation des couches sociales au nom desquelles était en principe mené le combat. Mais toute lutte prolongée contre la violence fait surgir le problème du renforcement des législations répressives (pouvoirs spéciaux en Algérie 1956, loi française anti-casseurs 1972, aujourd'hui abrogée); surtout elle alourdit le climat politique (les fameuses "années de plomb" en Allemagne) et réactive les discours d'ordre ou les exigences sécuritaires qui constituent des demandes de contrôle social accru. Cependant la violence n'est pas condamnée à disparaitre. Pour deux raisons au moins. Tout d'abord parce que les normes démocratiques elles-mêmes inclinent à une tolérance accrue des modalités d'expression contestataires, y compris lorsqu'elles empruntent la forme d'une violence qui demeure soft. Il arrive de plus en plus souvent à l'Etat de supporter un certain niveau de violence, pendant un certain temps; qu'il s'agisse de laisser ouverte la possibilité d'une négociation (le conflit Comapêche en septembre 1991) ou simplement de donner à une frustration collective une sorte d'expression purgative (le conflit des chauffeurs routiers en juillet 1992). Autre facteur qui incline à sa persistance : le fait que la forclusion générale du recours à la violence physique l'érige, en retour, en figure emblématique de la transgression. Et cela d'abord dans les groupes sociaux menacés économiquement et plus faiblement acculturés aux valeurs dominantes. D'où les troubles des banlieues, ou encore l'évolution de certains conflits sociaux dans l'agriculture et les industries économiquement déclinantes. Mais la volonté de transgression touche aussi des 
individus dont l'itinéraire biographique est dominé par le refus de toute intégration ; ils constituent un vivier pour d'éventuelles organisations clandestines. Les sociétés occidentales ont sans doute un long chemin à parcourir en compagnie de radicaux en quête de "justes causes". La violence politique est réputée déstabilisatrice. En réalité, ce jugement spontané n'épuise pas tous les aspects d'un phénomène plus complexe. Il est probable au contraire que les démocraties pluralistes tirent quelque profit d'un certain niveau de violence dans deux catégories de situations. C'est d'abord celle d'une menace, externe ou interne, qui manifeste l'existence d'un ennemi irréductible ; il sera possible de l'invoquer pour susciter un resserrement des allégeances aux valeurs de l'Etat et de l'ordre démocratique - encore faut-il que celle-ci demeure suffisamment marginale pour pouvoir être contenue sans mise en place de mesures exceptionnelles durables dont l'effet serait au contraire de gangrener la démocratie - . C'est aussi, dans une toute autre direction, la violence chronique de (relativement) faible intensité qui, fréquemment, se déploie dans les conflits sociaux. En dehors des canaux institutionnels mais en connexion étroite avec eux, elle contribue objectivement à élargir l'expression politique de groupes socioprofessionnels largement démunis de ressources strictement politiques. Il n'est donc pas inexact de dire que, dans ce cas de figure, elle permet paradoxalement d'enrichir la démocratie ${ }^{74}$.

\section{NOTES}

1. Guerre de Jugurtha. III. Historiens romains, Gallimard, 1984, t.I. p. 670.

2. J.P. Sartre, Préface à Frantz Fanon. Les damnés de la terre, (1961), 2 éd. Maspéro, 1968.

3. G. Sorel, qui déteste J. Jaurès, observe avec acrimonie que celui-ci, malgré son humanisme, absout les massacres de septembre 1792 in Réflexions sur la violence, (1908), Rééd, Seuil, 1990, p.105.

4. Voir la banque de données créée à l'Institut Français de Polémologie sous la responsabilité de Daniel Hermant.

5. T. Gurr, Why Men Rebel, Princeton University Press, 1970 ; I. Feierabend, R. Feierabend (Eds), Anger, Violence and Politics. Theories and Research, Englewood Cliffs, Prentice Hall, 1972 ; D. Hibbs, Mass Political Violence. A cross-national causal analysis, New York, Wiley, 1973 ; E. Muller "A Test of Partial Theory of Political Violence", American Political Science Review, 1972, III, p.928 et ss.

6. Pour une mise au point récente, P. Bourdieu, Réponses, Seuil, 1992, p.116 et ss. 7. "A Structural Theory of Aggression" in I. Feierabend, R. Feierabend, T. Gurr (Eds), Anger, Violence and Politics, op. cit. p.85.

8. Violence et politique, Gallimard, 1978, p.101.

9. Loc. cit. p.102.

10. Les règles de la méthode sociologique, (1895), Rééd, PUF, 1990, p.37.

11. La dynamique de l'Occident, (1939), Rééd, Calmann-Lévy, 1990, p.198.

12. Outre les ouvrages cités dans la note 4 , voir également J. W. Lapierre, "La violence dans les conflits sociaux", in Centre d'Etudes de la civilisation contemporaine, Desclée 
de Brouwer, 1968 ; E. Muller, Aggressive Political participation, Princeton University Press, 1979 ; J. C. Chesnais, Histoire de la violence en Occident de 1800 à nos jours, R. Laffont, 1981 ; C. Tilly, La France conteste. De 1600 à nos jours, (Trad), Fayard, 1986. Cf. également les enquêtes empiriques de $\mathrm{E}$. Weede et $\mathrm{M}$. Midlarski citées précédemment. 13. A. Oberschall, Social Conflict and Social movements, Englewood Cliffs, Prentice Hall, 1973, p.133 ; E. Zimmermann, Political Violence, Crises and Revolutions. Theories and Research, Cambridge (Mass) Shenkman, 1983, p.9.

14. Réflexions sur la violence, (1908), Réed, Seuil, 1990, p.169.

15. Political Violence. The Behavioral Process, New York, St Martin's Press, 1969, p.13.

16. P. Vidal-Naquet, La torture dans la République.1954-1962., Ed de Minuit, 1972 ; P. Taylor, Beating the Terrorists ? Interrogation in Omagh, Gough and Castlereagh, Harmondsworth, Penguin, 1980.

17. A. Corbin, "L'Histoire de la violence dans les campagnes françaises au XIXe siècle. Esquisse d'un bilan", Ethnologie française, 1991, T.III, p.224 et ss.

18. M. Wieviorka, La France raciste, Fayard,1992.

19. J. K. Galbraith, La paix indésirable ?, (1967), Trad., Calmann-Lévy , 1968, p.100.

20. Loc. cit. p.101.

21. D. Monjardet, "Le maintien de l'ordre. Technique et idéologie professionnelle des C.R.S", Déviance et Sociétés, 1988, n², p.101 et ss.

22. Carl. Schmitt, La notion de politique (1932), Trad., Flammarion, 1992, p.69.

23. Hemingway, dans “le récit de Pablo", montre bien comment, pendant la guerre d'Espagne, faire executer des propriétaires terriens par les paysans, est un moyen efficace de les identifier à un camp ; in Pour qui sonne le glas ?, Trad., Rééd., Gallimard, 1965, p.118 et ss.

24. B. Orfali. "L'homme de violence" in L'adhésion au Front National, Kimè,1990, p.194.

25. Une correspondance de F. Chipaux signalait l'adhésion de nombreux bosniaques musulmans au mouvement croate ultra : H.O.S. considéré par eux comme très "professionnel" ; et cela en dépit de ses positions hostiles à la communauté musulmane ; in Le Monde, 3 septembre1992. On peut aussi méditer l'itinéraire politique d'un Lacombe Lucien à partir du film de Louis Malle.

26. Par exemple sur l'emploi du mot "terroriste", D. Hermant, D. Bigo, "La relation terroriste : cadre sociologique pour une analyse comparatiste", Etudes polémologiques, 1988 T. III, p.52 et ss.

27. "Vie d'Alexandre", in Plutarque. Les vies des hommes illustres, Chap XXXI, Trad., Gallimard, 1968. t II p.343.

28. "La violence se manifeste lorsque le pouvoir est menacé...Parler d'un pouvoir non violent est en fait une tautologie". in Sur la violence, Trad., Rééd., Calmann-Lévy, 1972, p.157.

29. Political Violence, Crises and Revolutions...op. cit, p.157 et ss.

30. L. Coser, Les fonctions du conflit social, Trad., PUF, 1982, p.131 et 132.

31. Rechercher après coup qui a voulu la guerre, qui en est responsable, correspond à une exigence politique et morale, mais aussi au besoin d'exorciser l'angoisse d'être dominé par des processus sans sujet. Pourtant il n'est pas sûr qu'il existe une réponse satisfaisante dans tous les cas de figure.

32. F. Engels. Préface à Les luttes de classes en France, (1895), Rééd., Editions sociales, 1967, p.21 et ss ; G. Sorel, Reflexions sur la violence, op. cit. p.67. 
33. Le recrutement des C.R.S. s'opère d'ailleurs massivement dans les catégories ouvriers et paysans. Sur ce point, D. Monjardet. "Le maintien de l'ordre. Technique et idéologie professionnelle des C.R.S", art. précité.

34. P. Bourdieu, La Distinction, Ed. de Minuit, 1979, p.239.

35. Social Conflict and Social Movements, op. cit, p.332.

36. P. Champagne, "La manifestation comme action symbolique" in P. Favre (Dir). La manifestation, Presses de la F.N.S.P, 1990, p.339 et ss. Egalement, M. Offerlé, ibid, p.117. 37. Pour Etzioni, les manifestations sont un élément important du système politique démocratique. Cette thèse qui a sa justesse, est néammoins l'objet d'une critique argumentée de P. Favre in La manifestation, op. cit. p.61 et ss ; également, P. Favre, "Nature et statut de la violence dans les manifestations contemporaines", Cahiers de la Sécurité Intérieure, 1990, p.149 et ss.

38. Voir sur ce point I. Sommier, "C.G.T. Du service d'ordre au service d'accueil", Genèses, 1993, n 12 (à paraitre).

39. D. Apter, Pour l'Etat contre l'Etat, Economica, 1988, p.234. Sur la notion de moment disjonctif empruntée à Habermas, loc. cit. p.236.

40. S. Milgram, Soumission à l'autorité, (1972), Trad., Calmann-Lévy, 1974, p.194 et ss.

41. M. Wieviorka, D. Wolton, Terrorisme à la une : medias, terrorisme et démocratie, Gallimard, 1987.

42. M .Wieviorka, Sociétés et terrorismes, Fayard, 1988 ; R. Catanzaro (Ed), Terrorism and the Red Brigades in Italy, Londres, Pinter publishers, 1991 ; R. Dufour, "Les ressorts psychologiques de l'efficacité publicitaire du terrorisme", Etudes polémologiques, 1986, $n^{\circ} 1$, p.36.

43. B.Jenkins cité par Ph. Schlesinger, Media, State and Nation, Londres, Sage, 1991, p.

24.

44. H. Arendt, Le système totalitaire, Trad., Rééd., Seuil, 1972, p.209 et ss.

45. A. Mitscherlich, Le Deuil impossible, Trad., Payot, 1972 ; D. Bar On, L'héritage infernal. Des filles et fils de nazis racontent, Trad., Eshel, 1991. Voir également le personnage de Porfirio dans le "roman" de D. Fernandez. Porfirio et Constance, Grasset, 1991, p.255 et ss.

46. Parlant des troubles de 1848 à Paris, Engels emploie les termes suivants : "Le mouvement était là, instinctif, spontané, impossible à étouffer". Préface précitée p.18. 47. Sur ce point, P. Braud, Le jardin des délices démocratiques, Presses de la F.N.S.P, 1991, p.112 et ss.

48. La définition exacte de Gurr est : “Discrepancy between men's value expectations and their value capabilities, i.e. a discrepancy between the goods and conditions of life they believe as their due, and the goods and conditions they think they can in fact get and keep." in Why Men Rebel, Princeton University Press, 1971, p.319.

49. Ce point de vue classique est critiqué, sur la base de matériaux empiriques non compatibles, par E. Weede, "Some New Evidence on Correlates of Political Violence : Income Inequality, Regime Repressiveness and Economic Development" in European Sociological Review, 1987,III, p.97 et ss. Dans l'ouvrage dirigé par T. Gurr (Handbook of Political Conflict. Theory and Research, New York, Free Press, 1980) on trouvera à la fois une présentation synthétique de ces théories (H. Eckstein p.144 et ss) et une critique (E. Muller p.97 et ss).

50. R. Leveau, "Reflexions sur le non passage au terrorisme dans l'immigration clandestine en France" in Etudes Polémologiques, 1989, I, p.141; Voir également les travaux de S. Body-Gendrot (à paraitre). 
51. A. Bandura, Aggression. A social learning analysis, Englewood Cliffs, Prentice Hall, 1973, p.59.

52. Voir ci-après les contributions de N. Duclos, C. Journès et I. Sommier.

53. M. Pigenet, "La manifestation Ridgway du 28 mai 1952" in P. Favre (Dir), La manifestation, op. cit. p.261 et ss.

54. Essais sur la violence, Meridiens-Klincksieck, 1984. Cf. également les textes situationnistes de mai 1968 cités in A. Schnapp, P. Vidal-Naquet, Journal de la Commune étudiante, Seuil, 1968.

55. La violence et le sacré, Grasset, 1972, Rééd., Hachette, 1989, p.11.

56. Loc. cit, p.122.

57. Le bouc émissaire, Grasset, 1982, p.30.

58. Au sens retenu in Le jardin des délices démocratiques, op. cit, p.20 et p.245 et ss.

59. Cf. le témoignage de Hans Joachim Klein, La mort mercenaire,Trad., Seuil, 1980, p. 248.

60. C. Tilly, From Mobilization to Revolution, Reading, Addison-Wesley, 1978, p.201 et ss.

61. Voir ci-après les contributions de N. Duclos et I. Sommier.

62. La dynamique de l'Occident, op. cit. p.235 et ss.

63. "La grève générale syndicaliste offre les plus grandes analogies avec le premier système de guerre (considéré par lui comme admirable)...Le prolétariat a le sentiment très net de la gloire qui doit s'attacher à son rôle historique et de l'héroïsme de son attitude militante ; il aspire à l'épreuve décisive dans laquelle il donnera toute la mesure de sa valeur", Réflexions sur la violence, op. cit. p.164.

64. P. Anderson, Le marxisme occidental, Trad., Maspero, 1977. voir également L. Kolakowski, Histoire du marxisme, Trad., Fayard, 1987, t.II, p.562-563.

65. Il en va d'ailleurs également des "violences symboliques" auxquelles nous accoutument certaines pratiques de la société de communication : acharnement médiatique au mépris des droits des intéressés ou des conséquences subies par leurs proches ; production de stéréotypes dévalorisants. Par ailleurs la contribution ci-après de $C$. Haroche attire l'attention sur la violence des codes de bonnes manières et leur fonction éventuellement politique.

66. On opèrera ici une analogie avec le raisonnement de Pascal sur la justice : "Ne pouvant faire qu'il soit force d'obéir à la justice, on a fait qu'il soit juste d'obéir à la force ; ne pouvant fortifier la justice, on a justifié la force, afin que la justice et la force fussent ensemble, et que la paix fût, qui est le souverain bien". "Pensées" (238) in Oeuvres complètes, Gallimard, 1964, p.1152.

67. K. Lorenz, Essais sur le comportement animal, Trad., Seuil, 1970, p.137 et ss ; voir également P. Karli, L'homme agressif, Odile Jacob, 1987.

68. G. Moser, L'agression, PUF, 1987, p.115 et ss.

69. M. Wieviorka, D. Wolton, Terrorisme à la une, Gallimard, 1987, p.246.

70. Gurr, Why Men Rebel, op. cit. p.320.

71. M. Dubourdieu, B. Tarrin, "Evaluation des politiques de formation : l'exemple de la police nationale", Les Cahiers de l'IHESI ,1990 n² p.117 et ss. Egalement la contribution ci-après de $P$. Bruneteaux.

72. A la différence du livre de Merleau-Ponty (Humanisme et Terreur, 1948) beaucoup de textes ultérieurs (sartriens ou situationnistes) ont une signification surtout déclamatoire au sens où se creuse l'écart entre ce qui se joue avec les mots et ce qui se vit concrètement sur le terrain des luttes politiques, du moins après la guerre d'Algérie. 
73. Sur ce point, la communication ci-après d'A. Mercier. Il est arrivé que des journalistes se voient accusés de rapporter trop complaisamment les déclarations et activités de groupes clandestins combattus par l'Etat. Outre le cas corse étudié par X. Crettiez, voir les exemples cités in P. Schlesinger. Media, State and Nation, Londres, Sage, 1991, p.18, ainsi que l'ouvrage de D. Paletz et A. Schmid. Terrorism and the Media, Londres, Sage, 1992. Adde : D. Hermant, D. Bigo. "La relation terroriste" in Etudes polémologiques, 1988 III p.71.

74. Dans un sens assez voisin, l'auteur de The Theory of Stable Democracy, H. Eckstein, écrivait : "Le risque de conflits à bas niveau de violence est un prix que les démocraties doivent s'attendre à payer en contrepartie de la liberté préservée vis-à-vis de l'Etat et d'autres autorités sociales..." in H. Eckstein, T. Gurr. Patterns of Authority. A structural basis for political inquiry, New York, Wiley and Sons, 1975, p.452.

INDEX

Mots-clés : sociologie des conflits (polémologie), violence, sciences politiques 